\title{
5 UPV EHU \\ Exploiting superspace to enable DFT calculations of modulated structures with disordered sites \\ using the example of mullite
}

\section{Paul B. Klar, Xabier M. Aretxabaleta, Gotzon Madariaga}

Department of Condensed Matter Physics, University of the Basque Country UPV/EHU, Bilbao, Spain

\section{DFT of incommensurate, disordered structures seems impossible}

Density functional theory calculations (DFT) allow to investigate crystal structures and their properties including the electronic structure or elastic properties, but are limited to small structures (few hundred atoms) with fully occupied sites. Modulated structures with harmonic occupational modulations at first sight are not suitable for DFT. Here we describe an approach exploiting superspace [1] using the example for mullite [2].

\section{Approach to obtain an ordered commensurate approximation (O.C.A.)}
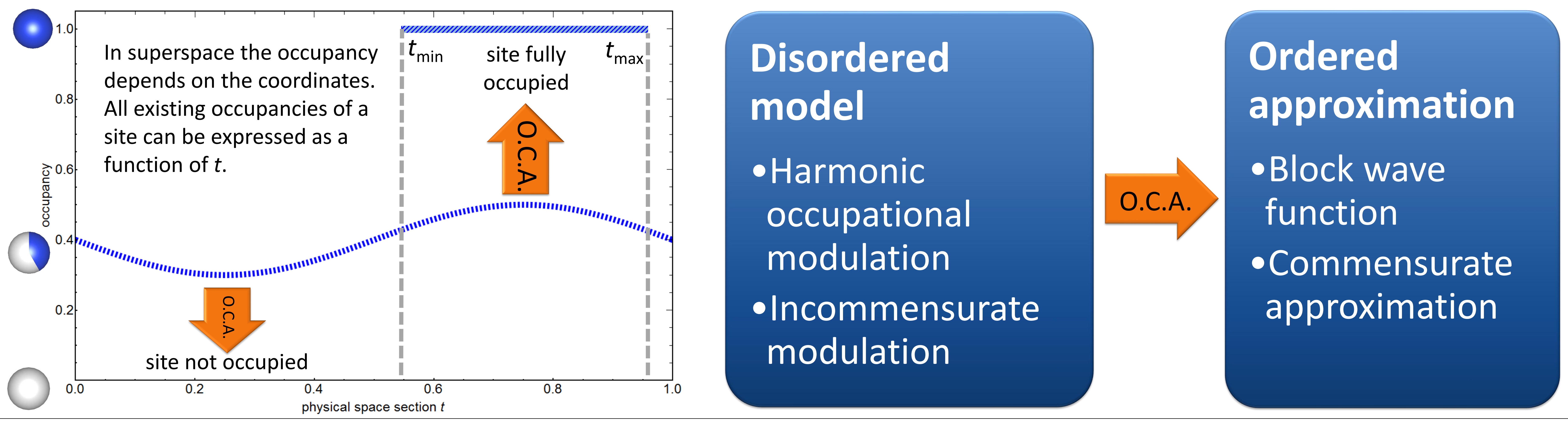

\section{Example: Al/Si ordering in disordered, modulated mullite not known}
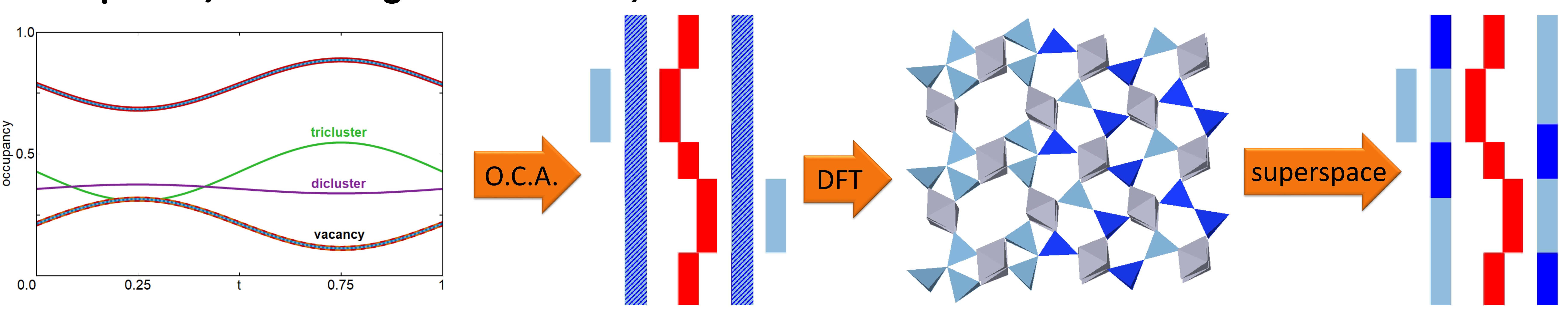

Using the above approach, an $\mathrm{Al} / \mathrm{Si}$ ordering pattern could be determined in physical space and in superspace. The ordering pattern of several different compositions results to be the same.

\section{Comparison with experimental Al/Si ordering}

$\mathrm{Al} / \mathrm{Si}$ ordering of a superspace model of mullite could not be refined directly, but could be derived from the modulation of the volumes of the tetrahedra [3]. These results are in good agreement with the Al/Si ordering determined with the above approach.

\begin{tabular}{|c|c|c|c|c|c|c|c|c|}
\hline cluster type & & Si-Si & Si-Al & Al-Si & Al-Al-Al* & Al*-Al-Al & Si-Al-Al & Al-Al-Si \\
\hline $\begin{array}{l}t \text { of highest probabil } \\
\text { (refined model) }\end{array}$ & & 0.75 & 0.13 & 0.37 & 0.60 & 0.90 & 0.63 & $0 . \varepsilon$ \\
\hline block wave function & $t_{\text {left }}$ & 0.7 & 0 & 0.35 & 0.5 & 0.85 & 0.65 & 0.8 \\
\hline (DFT model) & $t_{\text {right }}$ & 0.8 & 0.15 & 0.5 & 0.65 & 1 & 0.7 & 0.85 \\
\hline
\end{tabular}

\section{References}

[1] Pinheiro \& Abakumov, (2015), IUCrJ 2, 137

[2] Schneider et al. (2008), J Eur Cer Soc 28, 329

[3] Klar et al. (2018), IUCrJ, accepted

\section{Acknowledgment}

We thank the Spanish Government/ FEDER funds (MAT-2015-66441-P) and the Basque Government (IT-779-13, PhD grant) for financial support. 